\title{
Surfactant Mixtures at the Oil-Water Interface
}

\author{
Mario Campana ${ }^{1}$, John. R. P. Webster ${ }^{2}$, Thomas Gutberlet ${ }^{3}$, Kamil Wojciechowski ${ }^{*}$ \\ and Ali Zarbakhsh ${ }^{1 *}$
}

\begin{abstract}
${ }^{1}$ School of Biological \& Chemical Sciences, Queen Mary, University of London, Joseph Priestley Building, Mile End Road, London E1 4NS, United Kingdom.

${ }^{2}$ ISIS Neutron Facility, Science and Technology Facilities Council, Rutherford Appleton Laboratory, Harwell Science and Innovation Campus, Didcot, OX11 0QX, United Kingdom.

${ }^{3}$ Helmholtz-Zentrum Berlin für Materialien und Energie GmbH, Hahn-Meitner-Platz 1, D-14109 Berlin, Germany.

${ }^{4}$ Warsaw University of Technology, Faculty of Chemistry, Noakowskiego 3, 00-664 Warsaw, Poland.
\end{abstract}

*Authors to whom correspondence should be addressed. Email: a.zarbakhsh@qmul.ac.uk and kamil.wojciechowski@ch.pw.edu.pl 


\section{ABSTRACT}

We report the structural study of mixed monolayers of partially deuterated ,N'-di-hexadecyl(d33)-4,13-diaza-18-crown-6 ether (d-ACE16) and palmitic acid (PA) at the oil-water interface, in order to understand the mechanism of metal ion transport through Permeation Liquid Membrane (PLM) devices. The composition of the mixed monolayers remains constant with increasing spread amount and the saturation of the interface is achieved at a relatively low spread amount. The excess PA material is accommodated in the oil phase, playing an important role in equilibrating the interfacial concentration of ACE-16. The presence of PA increases the surface concentration of ACE-16 at low spread amount and facilitates its dissolution into the oil phase at the high spread amount. The result suggests a dynamic exchange between the bulk phase and the interface ensuring a continuous turnover which reflects their relevance in PLM devices. The conclusions regarding the role of a fatty acid in regulating the surface concentration of the alkylated azacrown ether and its dominant role in the bulk transport of metal ions through the membrane are consistent with the results of macroscopic studies reported earlier.

Graphical abstract

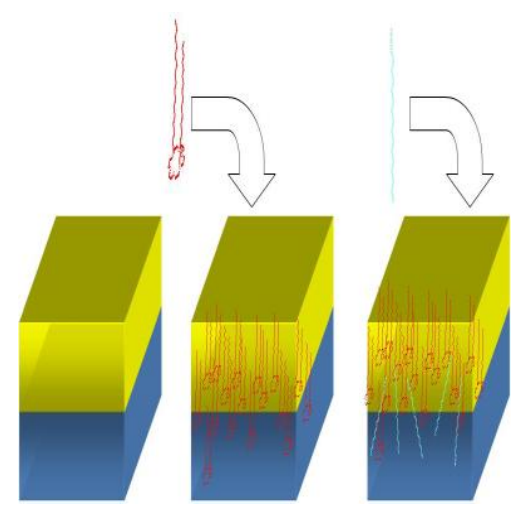

Highlights

- Neutron reflectivity at the oil-water interface. - Structural studies of mix surfactants at the oil-water interface. - Bulk transport of metal ions through a membrane. - The role of fatty acid in regulating surfactant surface concentration at interfaces.

Keywords

Oil-water interface; Azacrown ether; Permeation Liquid Membrane; palmitic acid. 


\section{INTRODUCTION}

Traces of heavy metal ions are present in natural waters under various forms, such as hydrated ions or complexes. Some of them are known to be toxic and can cause environmental damage. Their concentration in natural waters is usually very low, but it can significantly increase in polluted water [1].

Alkylated azacrown ethers (ACE) in combination with fatty acids have been extensively used as carriers for the transport of $\mathrm{Cu}(\mathrm{II}), \mathrm{Pb}(\mathrm{II}), \mathrm{Ni}(\mathrm{II})$ and $\mathrm{Cd}(\mathrm{II})$ ions in Permeation Liquid Membranes (PLM) for environmental analysis purposes [2]. A PLM device typically consist of two aqueous phases, called the source and strip solution, which are separated by a membrane soaked in an immiscible organic solvent [3]. The metal ions transport is based on selective complexation by a hydrophobic, membrane-soluble ligand (e.g., a mixture of ACE and fatty acid) dissolved in the organic solvent, which works as a shuttle between the two phases. The metal ions are released in the strip solution, where they are complexed with a hydrophilic, water-soluble ligand stronger than the carrier.

Azacrown ethers and their mixtures with fatty acids have been studied at the oil-water interface using surface-pressure isotherms and other classical surface characterization techniques [4]. However, the detailed structural information is still lacking. The isotherms obtained at the oil-water interface cannot be understood using a simple Langmuir or Frumkin model. The interfacial tension results indicate several possible orientations of the di-decyland di-hexadecyl-substituted ACE (ACE10 and ACE16) molecules at the water-oil (toluene) interface [5]. X-ray reflectivity carried out on these systems [6] has suffered from the lack of contrast to resolve these conformations and neutron reflectivity in combination with neutron contrast variation remains the most viable technique in resolving these structures.

As a first step in understanding these systems at oil-water interfaces and to provide a direct experimental evidence for possible conformation of ACE16 molecules alone, we measured a series of neutron reflectivity profiles at the buried oil-water interface. We found [7] that at the oil-water interface the azacrown ether molecules form a more diffuse extended layer compared to that at the air water interface. On the oil side the molecules were densely packed within a $17 \AA$ layer, possibly with the hydrophilic part of the molecule including the azacrown ether ring being immersed in the adjacent aqueous side of the interface. The latter consists of a thick $38 \AA$ dilute layer comprising staggered, loosely adsorbed (or aggregated) ACE16 molecules. With increasing the spread amount, the monolayer density increased at the oil side until the saturation at ca. $1.2 \times 10^{-6} \mathrm{~mol} \mathrm{~m}^{-2}$, above which the monolayer material was 
expelled to the aqueous side of the interface and ultimately dissolved into the adjacent bulk phases.

Next we studied spread monolayers of mixtures of deuterated N,N'-di-hexadecyl$\left(\mathrm{d}_{33}\right)$-4,13-diaza-18-crown-6 ether (d-ACE16) with different chain-length fatty acids (palmitic, stearic and hexacosanoic) using neutron reflectivity (NR) at the air-water interface [8]. By combining the surface pressure-area isotherm with NR measurements, the effect of the presence of fatty acids (FA) on the extent of the d-ACE16 monolayer dissolution at the air-water interface was quantified. Although all the fatty acids were capable of enhancing the retention of ACE16 at the interface, the optimum condition was achieved when the chain length of FA was matched to that of the ACE16 (i.e., $\mathrm{C}_{16}$, palmitic acid). These results provide an experimental justification for the current empirical composition of the carrier in the PLM membrane for metal ion transport, where the chain length of the fatty acid (dodecanoic acid) is closely matched to that of the azacrown ether (ACE10) [9]

In this paper we extended these studies to investigate the role of added fatty acid, in relation to the real PLMs at the oil-water interface, in order to closer mimic the composition of an ion-transporting membrane used in these devices. The primary question concerns the conformation of these ligands at the interface, which is investigated here.

\section{MATERIALS}

The h-ACE16 and d-ACE16, i.e. $N, N$ '-dihexadecyl-4,13-diaza-18-crown-6 ether $\left(\mathrm{C}_{44} \mathrm{H}_{70} \mathrm{~N}_{2} \mathrm{O}_{4}\right.$ and $\mathrm{C}_{44} \mathrm{H}_{24} \mathrm{D}_{66} \mathrm{~N}_{2} \mathrm{O}_{4}$, with hexadecyl chains deuterated) were synthesized by BDG Synthesis (BDG Synthesis, New Zealand [10]). Protonated palmitic acid (h-PA, $\mathrm{C}_{16} \mathrm{H}_{32} \mathrm{O}_{2}$ ) and deuterated palmitic acid (d-PA, 98\% D) were purchased from Aldrich. Hexadecane was also purchased from Aldrich and was purified by passing through an alumina column seven times prior to use. Hexadecane- $\mathrm{d}_{34}$ was obtained from Cambridge Isotope Laboratories (> 98\% D) and was used without further purification. All solutions and isotopic mixtures were prepared by mass. $\mathrm{D}_{2} \mathrm{O}$ was obtained from Fluorochem $(>99.9 \%$ atom D), and ultrapure $\mathrm{H}_{2} \mathrm{O}$ was produced using an Elgastat water purification unit. Contrastmatched silicon water contains approximately 38\% $\mathrm{D}_{2} \mathrm{O}$ (CMSi water); contrast-matched silicon hexadecane contains approximately $35 \%$ hexadecane-d mixed with h-hexadecane (CMSi oil). 


\section{EXPERIMENTAL}

The neutron reflectivity spectra were measured using the time-of-flight reflectometer FIGARO at ILL, Grenoble, France [11]. The reflectivity profiles were measured at $3.78^{\circ}$ providing a wide Q-range. The sample was under illuminated with a constant resolution $\delta Q / Q \sim 5.0 \%$.

Both the ACE16 and palmitic acid solutions were prepared separately in distilled chloroform. 1:1 mixtures of ACE16 and palmitic acid were then prepared prior to the spreading. Our recently developed experimental procedure for the study of amphiphiles at the fluid-fluid interface [7,12] was deployed. All measurements were conducted at $\mathrm{T}=298 \pm 0.5 \mathrm{~K}$ and the temperature was kept constant by means of a circulating water bath. The attenuation of a neutron beam upon transmission through an oil layer has been minimized by using a thin $(\sim 2.1 \mu \mathrm{m})$ oil layer film.

In the first part of the experiment, both the oil and water were contrast-matched to silicon $\left(\mathrm{Nb}=2.07 \times 10^{-6} \AA^{-2}\right)$. Initially, palmitic acid monolayers at the oil-water interface were studied as a reference; deuterated palmitic acid was used for this purpose. The data referring to ACE16 monolayers presented here are taken from [7]. Three contrasts, with different combinations of palmitic acid and ACE16 (d-PA with d-ACE16, h-PA with h-ACE16, h-PA with d-ACE16), were used to resolve the conformation of both species. Two additional contrasts, which will be discussed later, were used to provide extra sensitivity to the conformation at the interface. The full contrast scheme used is outlined in Table 1.

Table 1. Detailed summary of the five contrasts deployed to calculate adsorbed amount for both ACE16 and PA

\begin{tabular}{|c|c|c|c|c|c|}
\hline & Contrast 1 & Contrast 2 & Contrast 3 & Contrast 4 & Contrast 5 \\
\hline Oil phase & CMSi oil & CMSi oil & CMSi oil & CMSi oil & CMSi oil \\
\hline ACE16 & d-ACE16 & h-ACE16 & d-ACE16 & h-ACE16 & d-ACE16 \\
\hline PA & d-PA & h-PA & h-PA & h-PA & h-PA \\
\hline Water phase & CMSi water & CMSi water & CMSi water & $\mathrm{D}_{2} \mathrm{O}$ & $\mathrm{D}_{2} \mathrm{O}$ \\
\hline
\end{tabular}


The reflectivity profiles for Contrasts 1 and 3 were fitted to a single layer model with thickness $d$, scattering length density $N b$ and no roughness. A two layer model was necessary to fully represent Contrasts 2, 4 and 5 profiles.

The fitted $N b$ values can be written as a sum of the contributions from each of the four species representing the layer: ACE16, PA, oil, and water. When the adsorbed amount is determined by neutron reflectivity at the air-water interface, generally the water phase is contrast-matched to air (null reflecting water) so that the contribution of both species to the fitted $N b$ is equal to zero $\left(b_{a i r}=b_{n r w}=0\right)$ [13]. When oil and water are contrast-matched to silicon, the contribution from the two liquid phases must be taken into account, hence the total $\mathrm{Nb}$ of the layer is written as:

$$
N b_{\text {layer }}=N_{A C E 16} b_{A C E 16}+N_{P A} b_{P A}+N_{\text {oil }} b_{\text {oil }}+N_{\text {water }} b_{\text {water }}
$$

Since the oil and water phases have the same scattering length density, their contribution to the $N b_{\text {layer }}$ can be grouped in:

$$
(N b)_{l i q}=N_{o i l} b_{o i l}+N_{\text {water }} b_{\text {water }}
$$

It must be stressed that $(\mathrm{Nb})_{\text {liq }}$ is the contribution of the two liquids to $N b_{\text {layer }}$ and not the scattering length density of bulk oil or water (i.e. it is a function of their volume fractions in the layer). Since we do not seek to determine the amount of oil and water in the adsorbed layer, equation 1 can be rewritten as:

$N b_{\text {layer }}=N_{A C E 16} b_{A C E 16}+N_{P A} b_{P A}+(N b)_{\text {liq }}$

The number density $N$ for ACE16 and PA was calculated by simultaneously solving the above equation for three different contrasts, 1-3. These were then used to calculate the adsorbed amount $\Gamma$ for both species using the following formula:

$\Gamma=N_{i} \frac{d}{N_{A}}$

where $d$ is the layer thickness, and $\mathrm{N}_{\mathrm{A}}$ is Avogadro's number.

In order to precisely ascertain the conformation of the adsorbed layer at the oil-water interface, two additional contrasts (4 and 5) were deployed (Table 1). For Contrast 4 we decided to use h-ACE16, h-PA and oil contrast-matched to silicon as in Contrast 2, but this 
time we used $\mathrm{D}_{2} \mathrm{O}$ as water sub-phase. Similarly, Contrast 5 is analogous to Contrast 3 (dACE16 and h-PA) with $\mathrm{D}_{2} \mathrm{O}$ as the aqueous sub-phase.

In a previous neutron reflectivity experiment we found that for the spread amount $\geq 4.50 \times 10^{-6} \mathrm{~mol} \mathrm{~m}^{-2}$ the ACE16 monolayers collapsed [7]. For this reason, the spread amount of the ACE16 and PA mixture was kept below this value at all times. The investigated spread amount of the individual components ranged from 0.80 to $3.20 \times 10^{-}$ ${ }^{6} \mathrm{~mol} \mathrm{~m}{ }^{-2}$. The same range of spread amount was also adopted for the analysis of PA alone at the oil-water interface.

\section{RESULTS}

Neutron reflectivity profiles for d-PA at the oil-water interface for spread amount ranging from 0.80 to $3.20 \times 10^{-6} \mathrm{~mol} \mathrm{~m}^{-2}$ are shown in Figure 1 . The fits to the profiles are shown by solid lines. All the profiles were fitted to a single block model with a thickness of $26 \pm 2 \AA$. The fitted parameters, layer thickness $(d)$ and scattering length density $(N b)$ are given in Table 2. No significant changes are observed in the reflectivity profiles with increasing spread amount for d-PA. To visualise the lack of significant changes in the reflectivity profiles, the un-shifted profiles are shown in Figure 1 insert. 


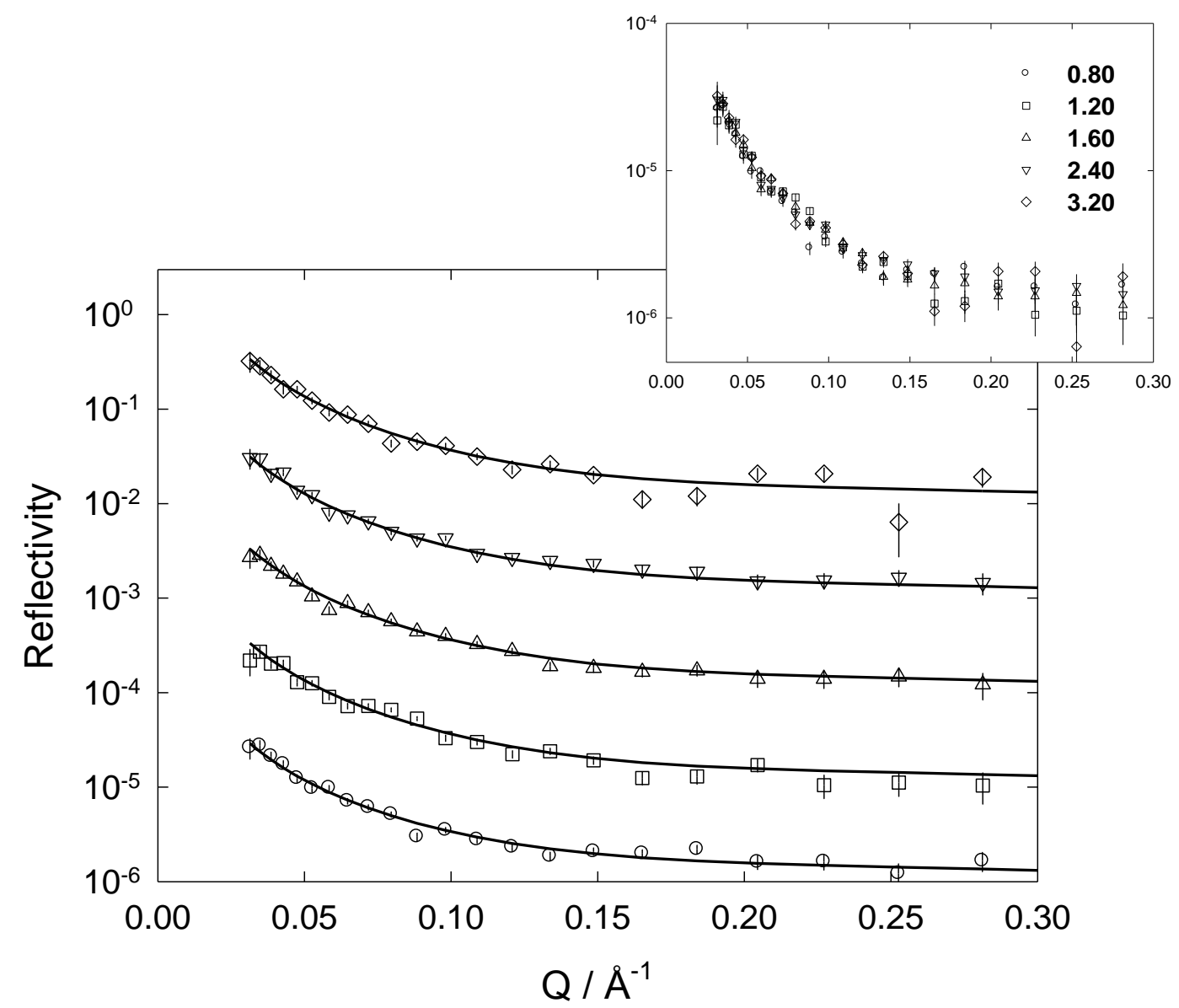

Figure 1. Reflectivity profiles for a series of d-PA spread amount at the CMSi oil-CMSi water interface. Solid lines correspond to the one layer fit to the data, the fitted parameters are given in Table 2. Profiles are shifted by a factor of $\times 10$ for the purpose of clarity. The un-shifted profiles are shown in figure insert to highlight the lack of significant differences. Labels for the spread amount are in figure insert (units: $\times 10^{-6}$ mol $\mathbf{m}^{-2}$ ).

Table 2. Parameters used for one layer fit to the reflectivity profiles shown in Figure 1 for d-PA at the hexadecane-water interface. Both oil and water are contrast-matched to silicon.

\begin{tabular}{|c|c|c|c|c|c|c|c|}
\hline \multirow[t]{2}{*}{$\begin{array}{l}\text { Spread amount } \\
\times 10^{-6} \mathrm{~mol} \mathrm{~m}^{-2} \\
\end{array}$} & & 0.80 & 1.20 & 1.60 & 2.40 & 3.20 & \multirow[b]{2}{*}{ Roughness / §̊ } \\
\hline & $d / \AA( \pm 2)$ & \multicolumn{5}{|c|}{$N b \times 10^{-6} / \AA^{-2}$} & \\
\hline Oil & I & \multicolumn{5}{|c|}{2.07} & 1 \\
\hline d-PA & 26 & 2.59 & 2.63 & 2.63 & 2.62 & 2.63 & 0.0 \\
\hline Water & I & \multicolumn{5}{|c|}{2.07} & 0.0 \\
\hline
\end{tabular}

For the ACE16 and PA mixture at the oil-water interface, three contrasts with both the oil and water contrast-matched to silicon were required to calculate the adsorbed amount for the 
individual components simultaneously. The reflectivity profiles for Contrast 1 and Contrast 3 for spread amount ranging from 0.80 to $3.20 \times 10^{-6} \mathrm{~mol} \mathrm{~m}^{-2}$ are shown in Figure 2 and Figure 3. Solid lines correspond to the fit to the data adopting a single layer model with no roughness. A layer thickness $d=30 \pm 2 \AA$ was found for spread amount of $2.40 \times 10^{-6} \mathrm{~mol} \mathrm{~m}^{-}$ ${ }^{2}$. The layer thickness increased to $34 \pm 2 \AA$ for the highest spread amount $\left(3.20 \times 10^{-6} \mathrm{~mol} \mathrm{~m}^{-}\right.$ ${ }^{2}$ ). Figure 2 insert and Figure 3 insert highlight how very little differences were observed within the reflectivity profiles as a function of increasing spread amount.

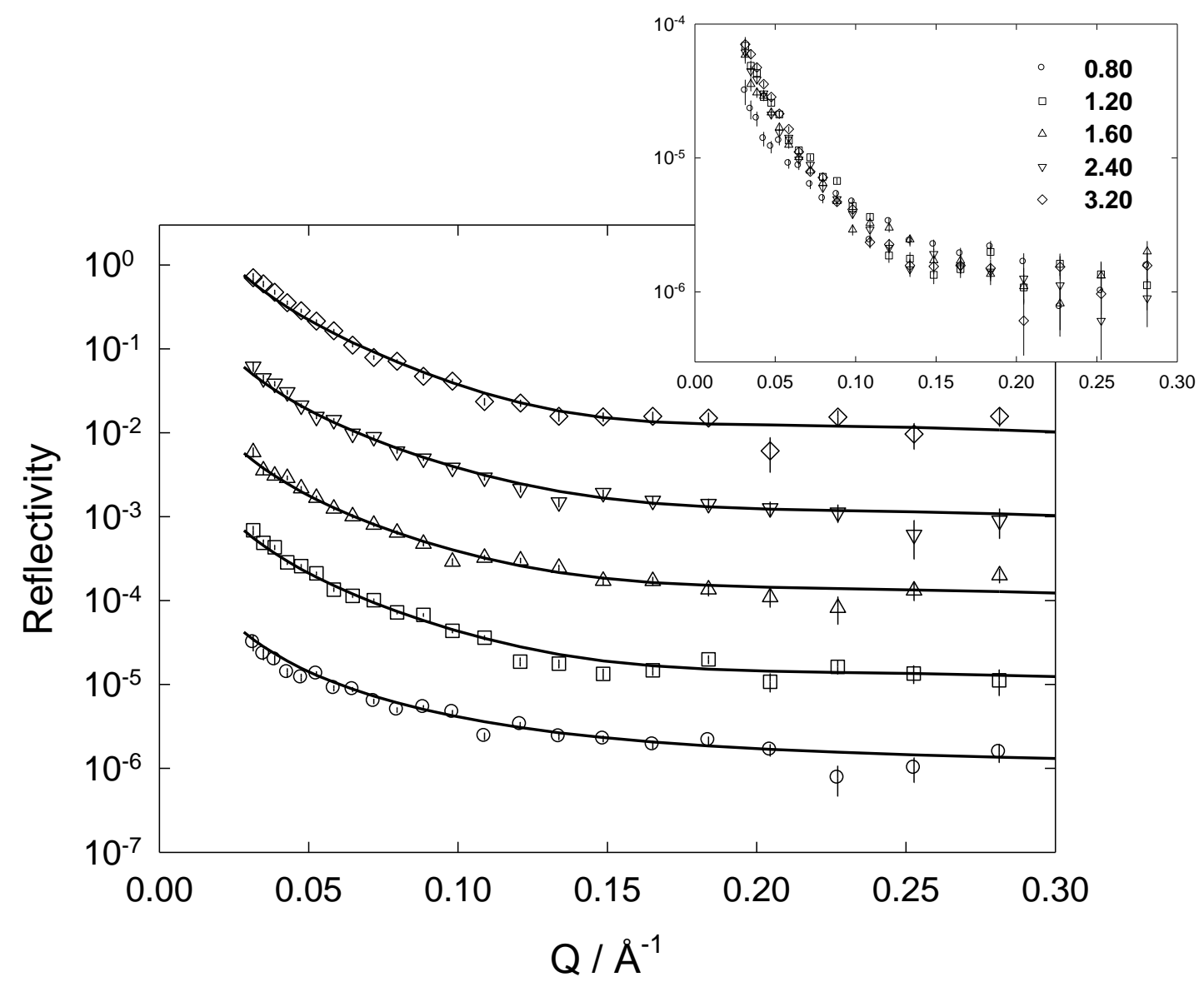

Figure 2. Reflectivity profiles for a series of d-ACE16 and d-PA spread amount (Contrast 1) at the CMSi oil-CMSi water interface. Solid lines correspond to the one layer fit to the data, the fitted parameters are given in Table 3 and Table 6. Profiles are shifted by a factor of $\times \mathbf{1 0}$ for the purpose of clarity. The un-shifted profiles are shown in figure insert to highlight the lack of significant differences. Labels for the spread amount are in figure insert (units: $\times 10^{-6} \mathrm{~mol} \mathrm{~m}^{-2}$ ). 


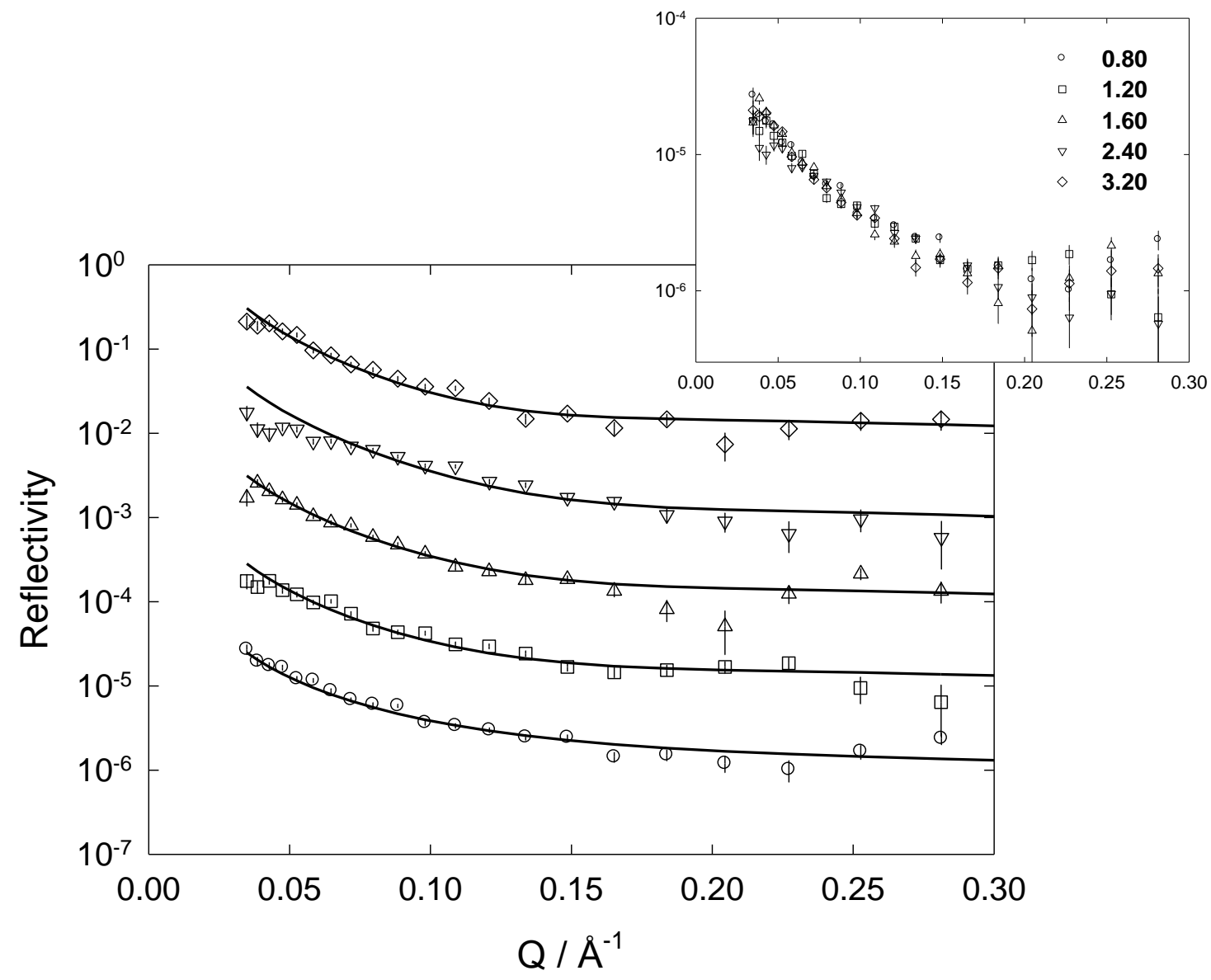

Figure 3. Reflectivity profiles for a series of d-ACE16 and h-PA spread amount (Contrast 3) at the CMSi oil-CMSi water interface. Solid lines correspond to the one layer fit to the data, the fitted parameters are given in Table 5 and Table 6. Profiles are shifted by a factor of $\times \mathbf{1 0}$ for the purpose of clarity. The un-shifted profiles are shown in

figure insert to highlight the lack of significant differences. Labels for the spread amount are in figure insert (units: $\times 10^{-6} \mathrm{~mol} \mathrm{~m}^{-2}$ ).

As opposed to Contrast 1 and Contrast 3, the one layer model for Contrast 2 requires a much smaller layer thickness ( $d=21 \pm 2 \AA$ for all five spread amounts). This suggests that the one layer model is not sufficient to adequately represent the interfacial region. Therefore we decided to apply a two layer model to fit the reflectivity curves for Contrast 2 . When the number of layers is increased, the number of fitting variables is also increased; hence in order to reduce the ambiguity in the fitting procedure the following constraints were applied:

(i) The overall layer thickness for all spread amounts was kept the same as observed for Contrast 1 and Contrast 3. 


$$
d_{\text {layer } 1}+d_{\text {layer } 2}=d_{C 1 \text { and } 3}
$$

Where $d_{\text {layer } 1}$ and $d_{\text {layer } 2}$ are the thicknesses of the two layers into which the interfacial region has been divided, whereas $d_{C l}$ and 3 is the thickness observed for the one layer fit to the reflectivity profiles for Contrasts 1 and 3.

(ii) The integrated scattering length density profile must give the same result as that for the one layer fit, i.e. constant adsorbed amount.

$$
\begin{gathered}
d_{1 \text { layer fit }} \times\left(N b_{\text {layer }}-N b_{b}\right) \\
=d_{\text {layer } 1} \times\left(N b_{\text {layer } 1}-N b_{b}\right)+d_{\text {layer } 2} \times\left(N b_{\text {layer } 2}-N b_{b}\right)
\end{gathered}
$$

Where $N b_{\text {layer }}$ is the scattering length density observed for the one layer fit to the profiles, $N b_{b}$ is the scattering length density of the liquid phases (both contrastmatched to silicon) and $N b_{\text {layer } 1}$ and $N b_{\text {layer } 2}$ are the scattering length densities of the first and second layer respectively.

The reflectivity profiles for Contrast 2 are shown in Figure 4; solid lines correspond to the two layer model fits.

The parameters adopted for the fits for Contrast 1, Contrast 2 and Contrast 3 are shown in Table 3, Table 4 and Table 5 for spread amount up to $2.40 \times 10^{-6} \mathrm{~mol} \mathrm{~m}^{-2}$. The fitting parameters for all the three contrasts for spread amount $3.20 \times 10^{-6} \mathrm{~mol} \mathrm{~m}^{-2}$, with layer thickness $d=34 \pm 2 \AA$ are grouped in Table 6 . 


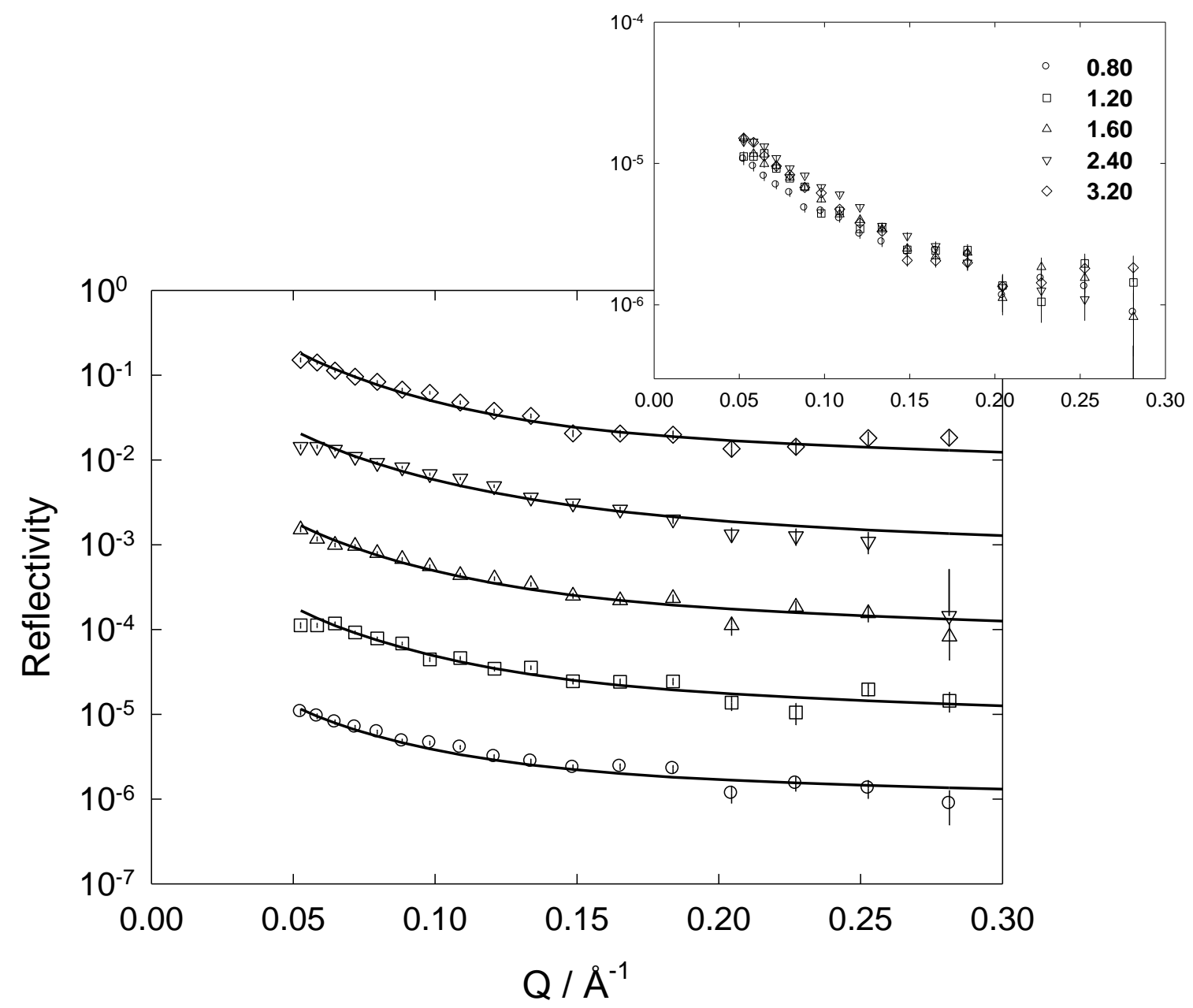

Figure 4. Reflectivity profiles for a series of h-ACE16 and h-PA spread amount (Contrast 2) at the CMSi oil-CMSi water interface. Solid lines correspond to the two layer fit to the data, the fitted parameters are given in Table 4 and Table 6. Profiles are shifted by a factor of $\times \mathbf{1 0}$ for the purpose of clarity. The un-shifted profiles are shown in

figure insert to highlight the lack of significant differences. Labels for the spread amount are in figure insert (units: $\times 10^{-6} \mathrm{~mol} \mathrm{~m}^{-2}$ ).

Table 3. Fitted parameters for the reflectivity profiles for Contrast 1 (Figure 2). Both oil and water are contrast-matched to silicon. The fitted parameters relative to the highest spread amount are given in Table 6.

\begin{tabular}{|c|c|c|c|c|c|c|}
\hline \multirow[t]{2}{*}{$\begin{array}{c}\text { Spread amount } \\
\times 10^{-6} \mathrm{~mol} \mathrm{~m}^{-2} \\
\end{array}$} & & 0.80 & 1.20 & 1.60 & 2.40 & \multirow[b]{2}{*}{ Roughness / Å } \\
\hline & $d / \AA( \pm 2)$ & \multicolumn{4}{|c|}{$N b \times 10^{-6} / \AA^{-2}$} & \\
\hline Oil & l & \multicolumn{4}{|c|}{2.07} & l \\
\hline d-ACE16, d-PA & 30 & 2.58 & 2.72 & 2.66 & 2.67 & 0.0 \\
\hline Water & 1 & \multicolumn{4}{|c|}{2.07} & 0.0 \\
\hline
\end{tabular}


Table 4. Fitted parameters for the reflectivity profiles for Contrast 2 (Figure 4). Both oil and water are contrast-matched to silicon. The fitted parameters relative to the highest spread amount are given in Table 6.

\begin{tabular}{|c|c|c|c|c|c|c|}
\hline $\begin{array}{c}\text { Spread amount } \\
\times 10^{-6} \mathrm{~mol} \mathrm{~m}^{-2}\end{array}$ & & 0.80 & 1.20 & 1.60 & 2.40 & \\
\hline & $d / \AA( \pm 2)$ & \multicolumn{4}{|c|}{$N b \times 10^{-6} / \AA^{-2}$} & Roughness / Å \\
\hline Oil & I & \multicolumn{4}{|c|}{2.07} & I \\
\hline \multirow{2}{*}{ h-ACE16, h-PA } & 15 & 0.89 & 1.04 & 1.04 & 1.20 & 0.0 \\
\hline & 15 & 1.95 & 1.95 & 1.95 & 1.98 & 0.0 \\
\hline Water & I & \multicolumn{4}{|c|}{2.07} & 0.0 \\
\hline
\end{tabular}

Table 5. Fitted parameters for the reflectivity profiles for Contrast 3 (Figure 3). Both oil and water are contrast-matched to silicon. The fitted parameters relative to the highest spread amount are given in Table 6.

\begin{tabular}{|c|c|c|c|c|c|c|}
\hline \multirow[t]{2}{*}{$\begin{array}{c}\text { Spread amount } \\
\times 10^{-6} \mathrm{~mol} \mathrm{~m}^{-2} \\
\end{array}$} & & 0.80 & 1.20 & 1.60 & 2.40 & \multirow[b]{2}{*}{ Roughness / A } \\
\hline & $d / \AA( \pm 2)$ & \multicolumn{4}{|c|}{$N b \times 10^{-6} / \AA^{-2}$} & \\
\hline Oil & l & \multicolumn{4}{|c|}{2.07} & l \\
\hline d-ACE16, d-PA & 30 & 2.56 & 2.57 & 2.60 & 2.64 & 0.0 \\
\hline Water & I & \multicolumn{4}{|c|}{2.07} & 0.0 \\
\hline
\end{tabular}

Table 6. Fitted parameters for the reflectivity profiles for Contrast 1, 2 and 3 for the highest spread amount $\left(3.20 \times 10^{-6} \mathrm{~mol} \mathrm{~m}^{-2}\right)$.

Contrast 1

d-ACE and d-PA

\begin{tabular}{|c|c|c|c|}
\hline & $\mathbf{d} / \mathbf{A}(\mathbf{\pm 2})$ & $\mathbf{N b} \times \mathbf{1 0}^{-\mathbf{6}} / \AA^{-\mathbf{2}}$ & Roughness $/ \mathbf{\AA}$ \\
\hline Oil & $/$ & 2.07 & $/$ \\
\hline $\mathrm{d}-\mathrm{ACE} 16, \mathrm{~d}-\mathrm{PA}$ & 34 & 2.68 & 0.0 \\
\hline Water & $/$ & 2.07 & 0.0 \\
\hline
\end{tabular}

Contrast 2

h-ACE and h-PA

\begin{tabular}{|c|c|c|c|}
\hline & $\mathbf{d} / \AA \mathbf{A} \mathbf{( 2 )}$ & $\mathbf{N b} \times \mathbf{1 0}^{-\mathbf{6}} / \AA^{-\mathbf{2}}$ & Roughness / \\
\hline Oil & $/$ & 2.07 & $/$ \\
\hline \multirow{2}{*}{ h-ACE16, h-PA } & 17 & 1.12 & 0.0 \\
\cline { 2 - 4 } & 17 & 1.95 & 0.0 \\
\hline Water & $/$ & 2.07 & 0.0 \\
\hline
\end{tabular}

Contrast 3

d-ACE and h-PA 


\begin{tabular}{|c|c|c|c|}
\hline & $\mathbf{d} / \AA \mathbf{A} \mathbf{( 2 )}$ & $\mathbf{N b} \times \mathbf{1 0}^{-\mathbf{6}} / \AA^{-\mathbf{2}}$ & Roughness $/ \AA$ \\
\hline Oil & $/$ & 2.07 & $/$ \\
\hline h-ACE16, h-PA & 34 & 2.54 & 0.0 \\
\hline Water & $/$ & 2.07 & 0.0 \\
\hline
\end{tabular}

To ascertain that the two layer model adopted for Contrast 2 is an adequate representation of all the three contrasts, the same model was adopted to fit the reflectivity profiles for Contrast 1 and Contrast 3. The two layer model could effectively represent the reflectivity profiles for all the contrasts (see Supporting information).

For the purpose of calculating the adsorbed amount, it is more convenient to use a one layer model as describe in the literature [14]. Therefore, for Contrast 2 the parameters for two layers need to be combined to give an equivalent single layer. It is known that the adsorbed amount $\Gamma$ is a function of the integrated area in the scattering length density profile [15]:

$$
\Gamma=\int_{-\infty}^{+\infty} N b(z) \mathrm{d} z
$$

This implies that a scattering length density profile consisting of two layers, each characterised by a certain $N b$ value, can be substituted with a different $N b$ profile, consisting of one layer only, as long as the integrated area is kept constant. This $N b$ value can be calculated using the weighted average, Equation 8, from the contribution of the two layers, whose values are shown in Table 4 and Table 6.

$$
N b_{\text {layer } 2}=\frac{d_{1} N b_{1}+d_{2} N b_{2}}{d_{1}+d_{2}}
$$

One should stress that this transformation is only valid when calculating the adsorbed amount at the interface, and not for structural determination of the adsorbed layer. The equivalent thickness and scattering length density values are given in Table 7. 
Table 7. The equivalent one-layer parameters for the reflectivity profiles for Contrast 2 , oil and water CMSi, h-ACE and h-PA. The $N b$ values are obtained as weighted average from the values relative to two layer model in Table 4 and Table 6.

\begin{tabular}{|c|c|c|c|c|c|c|}
\hline $\begin{array}{l}\text { Spread amount } \\
\times 10^{-6} \mathrm{~mol} \mathrm{~m}^{-2}\end{array}$ & & 0.80 & 1.20 & 1.60 & 2.40 & \\
\hline & $d / \AA( \pm 2)$ & \multicolumn{4}{|c|}{$N b \times 10^{-6} / \AA^{-2}$} & Roughness / Å \\
\hline Oil & I & \multicolumn{4}{|c|}{2.07} & I \\
\hline h-ACE16, h-PA & 30 & 1.42 & 1.50 & 1.50 & 1.59 & 0.0 \\
\hline Water & I & \multicolumn{4}{|c|}{2.07} & 0.0 \\
\hline
\end{tabular}

\begin{tabular}{|c|c|c|c|}
\hline $\begin{array}{c}\text { Spread amount } \\
\times \mathbf{1 0}^{-\mathbf{6}} \mathbf{~ m o l ~ m}^{-\mathbf{2}}\end{array}$ & $\boldsymbol{d} / \AA \mathbf{A} \mathbf{( \mathbf { 2 } )}$ & $\mathbf{N b} \times \mathbf{1 0}^{-\mathbf{6}} / \AA^{-\mathbf{2}}$ & Roughness $/ \AA$ \\
\hline Oil & $/$ & 2.07 & $/$ \\
\hline h-ACE16, h-PA & 34 & 1.54 & 0.0 \\
\hline Water & $/$ & 2.07 & 0.0 \\
\hline
\end{tabular}

It is noteworthy analysing in details the reflectivity profiles for Contrast 3. According to the Babinet's reciprocity principle, in a two phase system the scattering length densities of the two phases can be interchanged without affecting the scattering curve [16]. This implies that, considering negligible the reflectivity from the silicon-oil interface, two profiles such as those shown in Figure 5, presenting the same $\triangle N b$ with respect to the two liquid phases, are bound to have the same reflectivity profiles. 


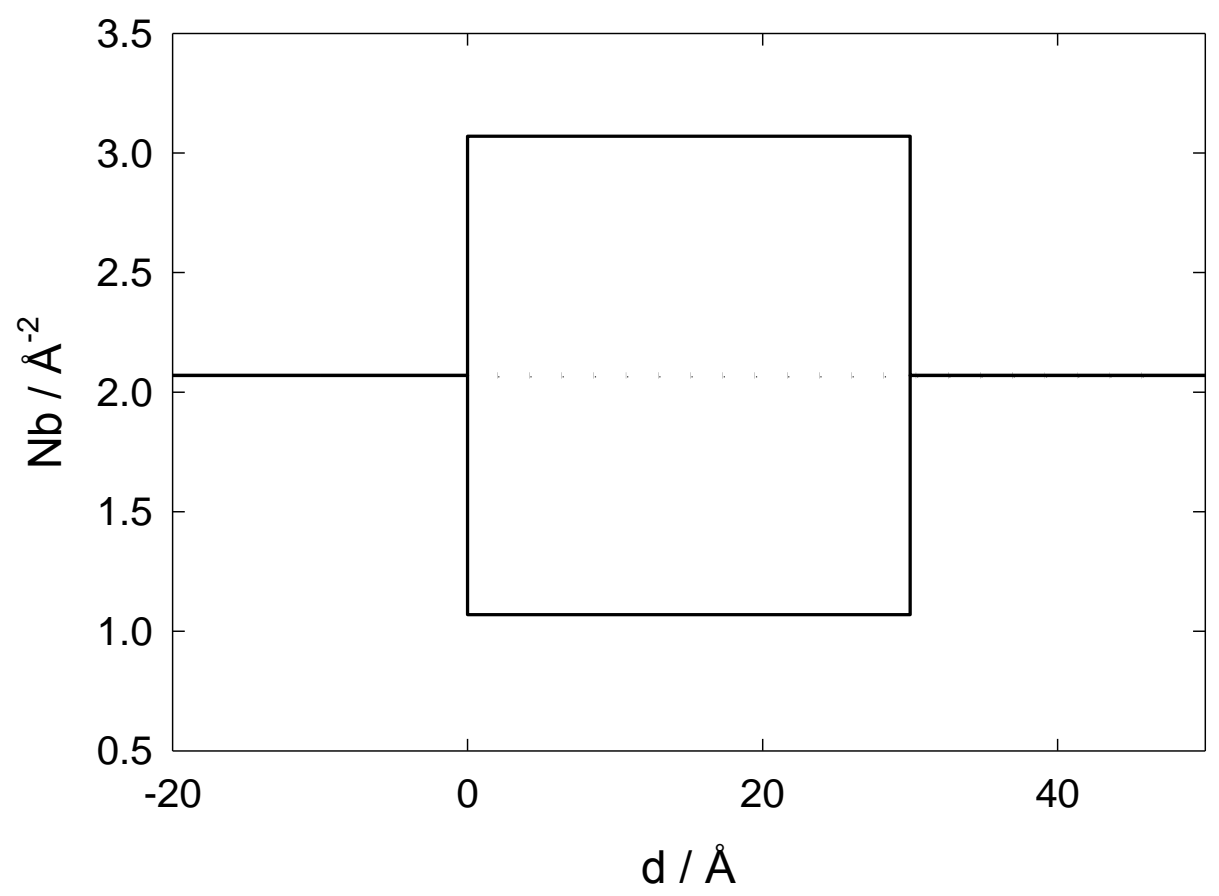

Figure 5. Schematic representation of two $N b$ profiles presenting $\Delta N b$ with same modulus between the adsorbed layer and the bulk phases. According to Babinet's reciprocity principle, such $N b$ profiles lead to identical reflectivity profiles.

In Contrast 1, where we have two deuterated species at the interface, one can with certainty assess that the $N b$ of the adsorbed layer will be higher than that of the silicon substrate. Likewise, in Contrast 2 two protonated species are adsorbed at the interface, and the $N b$ of the adsorbed layer will be lower than silicon. When both deuterated and protonated species are adsorbed at the interface, such as in Contrast 3, one cannot establish a priori whether the $\mathrm{Nb}$ of the adsorbed layer will be higher or lower than that of silicon. One way to assess whether the $\triangle N b$ between the adsorbed layer and the silicon will be positive or negative is to compare it to the contrasts where this has already been determined.

Let us consider the situation when the spread amount is $2.40 \times 10^{-6} \mathrm{~mol} \mathrm{~m}^{-2}$. The $\mathrm{Nb}$ values for Contrast 1 and Contrast 2 are summarised in Table 8, along with the $\Delta N b$ between silicon and the adsorbed layer in Contrast 3. 
Table 8. $\Delta N b$ between the adsorbed layer and the bulk phases for the three contrasts (spread amount $2.40 \times 10^{-6} \mathrm{~mol} \mathrm{~m}^{-2}$ ). Signs are omitted. The $N b$ of the adsorbed layer can be unequivocally assigned for Contrast 1 and 2, but this is not true for Contrast 3 where two situations are possible. However, we must discard the second possibility $\left(N b_{\text {layer }}=1.50 \times 10^{-6} / \AA^{-2}\right)$ : because of the presence of deuterated PA in the layer, $N b_{\text {layer }}$ for Contrast 3 cannot be lower than that for Contrast 2.

\begin{tabular}{|c|c|c|c|}
\hline \multicolumn{2}{|c|}{ Contrast } & $|\Delta N b| \times \mathbf{1 0}^{-\mathbf{6}} / \AA^{-2}$ & $N b_{\text {layer }} \times \mathbf{1 0}^{-\mathbf{6}} / \AA^{-2}$ \\
\hline $\mathbf{1}$ & d-ACE16 and d-PA & $\mathbf{0 . 6 0}$ & $\mathbf{2 . 6 7}$ \\
\hline $\mathbf{2}$ & h-ACE16 and h-PA & $\mathbf{0 . 4 8}$ & $\mathbf{1 . 5 4}$ \\
\hline $\mathbf{3}$ & d-ACE16 and h-PA & $\mathbf{0 . 5 7}$ & $\mathbf{2 . 6 4}$ \\
\hline
\end{tabular}

The adsorbed layer at the interface in Contrast 3 is composed of a mixture of d-ACE16 and hPA. If the d-ACE16 contribution to the reflectivity is stronger than h-PA contribution, the $N b_{\text {layer }}$ will be higher than that of silicon. On the other hand, if the contribution of h-PA is dominating, then the $N b_{\text {layer }}$ will be lower than silicon. It is important to note that in no case the $N b_{\text {layer }}$ for Contrast 3 , which contains some deuterated material (d-ACE16) can be higher than that observed for Contrast 1 (all deuterated) or lower than that for Contrast 2 (all hydrogenous). Hence, the only acceptable $N b_{\text {layer }}$ value for Contrast 3 is 2.64 . Should $\triangle N b$ be small enough that both possibilities were acceptable, one more contrast would be required to over constrain the calculations (for example, h-ACE16 and d-PA could be used with oil and water both contrast-matched to silicon).

The number densities for ACE16 and PA, $N_{A C E 16}$ and $N_{P A}$, obtained from the Contrasts 1-3 were used to calculate the adsorbed amount at the oil-water interface for the individual compounds using equation (4). In Figure 6(a) the adsorbed amount of ACE16 as a function of its spread amount at the oil-water interface in the presence and absence of PA are compared. The data referring to ACE16 alone are also shown [7]. An analogous comparison is shown for PA in Figure 6(b). The values for PA alone were measured in the first part of the experiment and refer to the reflectivity profiles in Figure 1. 


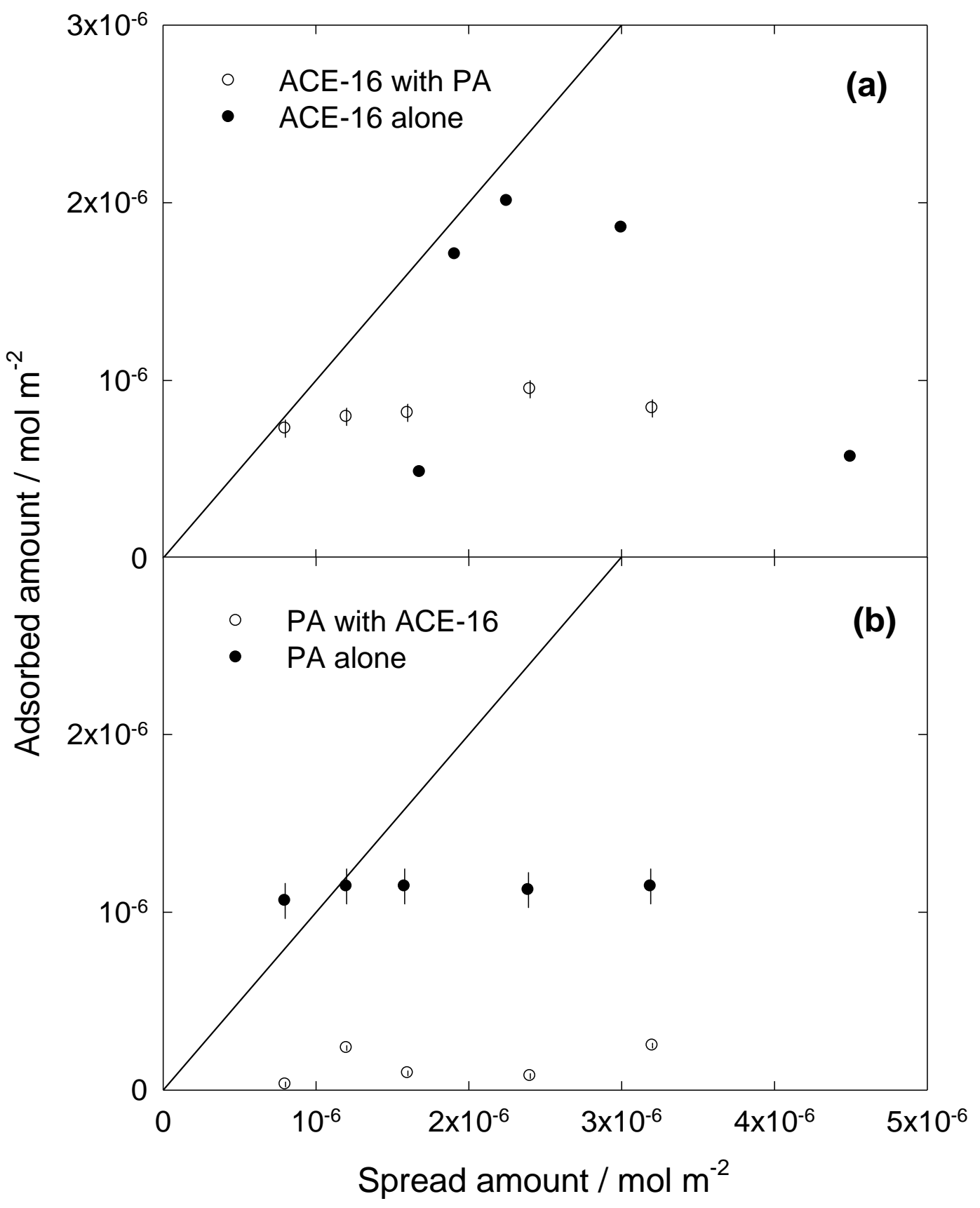

Figure 6(a): the adsorbed amount for ACE16 in presence $(\circ)$ and absence $(\bullet)$ of PA is presented. The straight line represents the maximum adsorbed amount at the interface assuming total retention at the interface. Figure 6(b): a similar representation is shown for PA alone (•) and PA in presence of ACE16 ( $)$.

The adsorbed amount for both species when they co-adsorb at the oil-water interface does not change significantly as a function of spread amount. This was not surprising for PA, where no changes were observed also when it was the only specie at the interface; however this steady adsorption was unexpected for ACE16. For both species there is generally a 
significant decrease in adsorbed amount when they move from being the only component at the interface to the condition when they are part of a mixture.

$\mathrm{D}_{2} \mathrm{O}$ was used as the aqueous sub-phase for Contrast 4 and Contrast 5 for the determination of the structural conformation of adsorbed layer. We have chosen $\mathrm{D}_{2} \mathrm{O}$ as the aqueous phase to maximise the difference in scattering length density between the two bulk phases, thus allowing the detailed structural characterisation of the adsorbed layer. The reflectivity profiles for Contrast 4 (h-ACE16 and h-PA at the contrast-matched silicon oil- $\mathrm{D}_{2} \mathrm{O}$ interface) and Contrast 5 (d-ACE16 and h-PA at the contrast-matched silicon oil- $\mathrm{D}_{2} \mathrm{O}$ interface) are shown in Figure 7 and Figure 8 respectively, solid lines correspond to the fit to the data. As expected, very few changes were observed in the reflectivity profiles with increasing spread amount. Such small changes are shown in Figure 7 insert and Figure 8 insert.

For Contrast 4, all the reflectivity profiles were found to be adequately represented by a two layer model consisting of a $28 \AA$ layer on the oil side of the interface and a rather diffuse $49 \AA$ layer on the aqueous side of the interface. The parameters for the fitting procedure are shown in Table 9. The same model used to represent the reflectivity profiles for Contrast 4 could only fit the profiles for Contrast 5 if an unreasonable value for interlayer roughness was used (roughness $>1 / 2 d$ ). With an exception of the roughness, similar parameters were used (Table 10): the thickness of the layer adjacent to the oil phase was kept constant between Contrast 4 and Contrast 5, whereas a slight reduction in thickness was observed for the layer in contact with the aqueous side of the interface (from $49 \AA$ to $42 \AA$ ). The $N b$ profiles for both Contrast 4 and Contrast 5 are shown in Figure 9. As small differences are observed for the profiles at different spread amounts, for each contrast only the average $N b$ values are shown for clarity. 


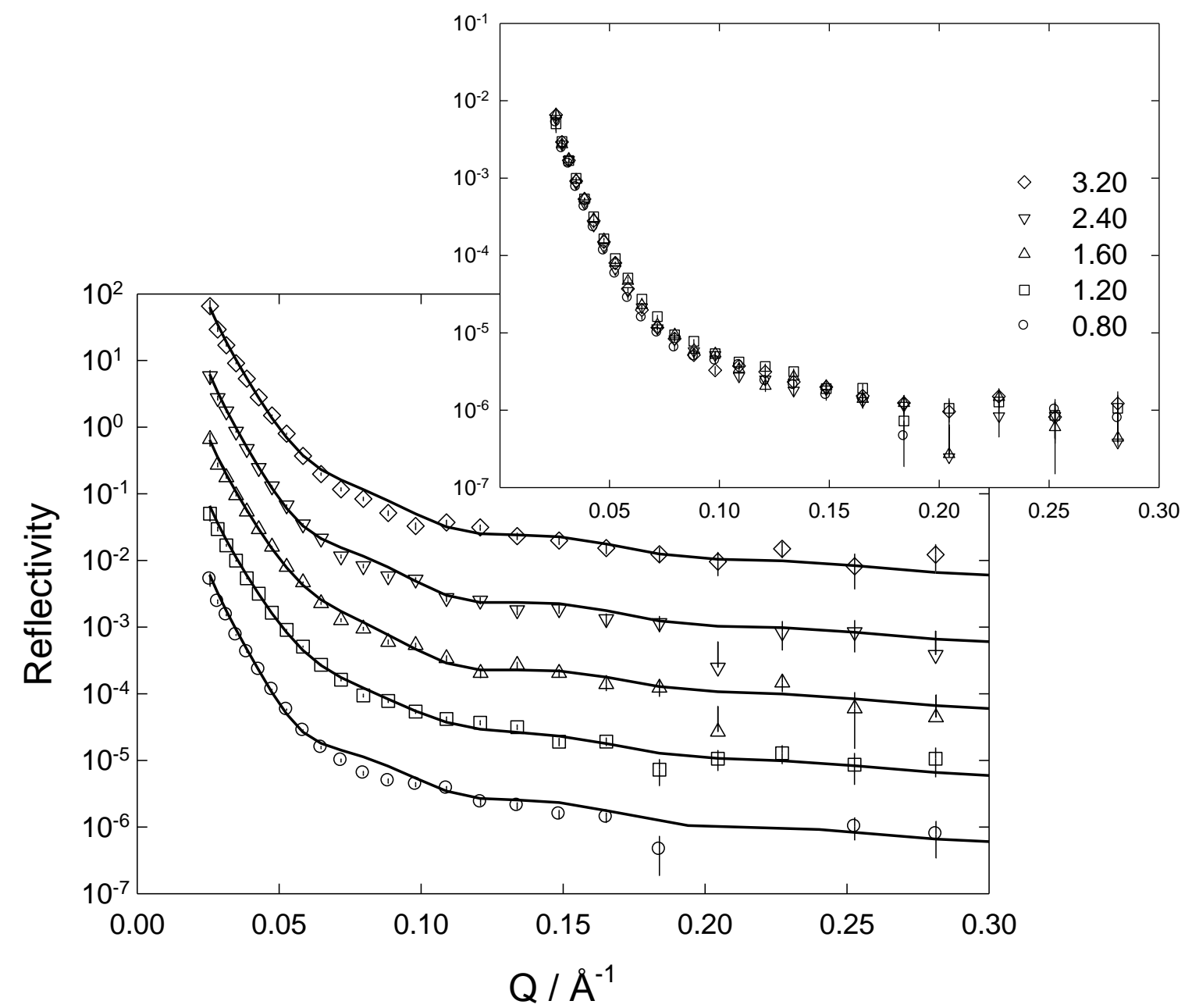

Figure 7. Reflectivity profiles for a series of h-ACE16 and h-PA spread amount (Contrast 4) at the CMSi oil- $\mathrm{D}_{2} \mathrm{O}$ interface. Solid lines correspond to the fit to the data, the fitted parameters are given in Table 9. Profiles are shifted by a factor of $\times 10$ for the purpose of clarity. The un-shifted profiles are shown in figure insert to highlight the lack of significant differences. Labels for the spread amount are in figure insert (units: $\times 10^{-6} \mathrm{~mol} \mathrm{~m}^{-2}$ ).

Table 9. Fitted parameters for the reflectivity profiles for Contrast 4 (Figure 7).

\begin{tabular}{|c|c|c|c|c|c|c|c|}
\hline $\begin{array}{l}\text { Spread amount } \\
\times 10^{-6} \mathrm{~mol} \mathrm{~m}^{-2}\end{array}$ & & 0.80 & 1.20 & 1.60 & 2.40 & 3.20 & \\
\hline & $d / \AA( \pm 2)$ & \multicolumn{5}{|c|}{$\mathrm{Nb} \times 10^{-6} / \AA^{-2}$} & Roughness / § \\
\hline Oil & I & \multicolumn{5}{|c|}{2.07} & I \\
\hline Layer 1 & 28 & 3.31 & 3.25 & 3.44 & 3.41 & 3.36 & 5.0 \\
\hline Layer 2 & 49 & 5.38 & 5.61 & 5.61 & 5.48 & 5.54 & 5.0 \\
\hline $\mathrm{D}_{2} \mathrm{O}$ & I & \multicolumn{5}{|c|}{6.35} & 5.0 \\
\hline
\end{tabular}




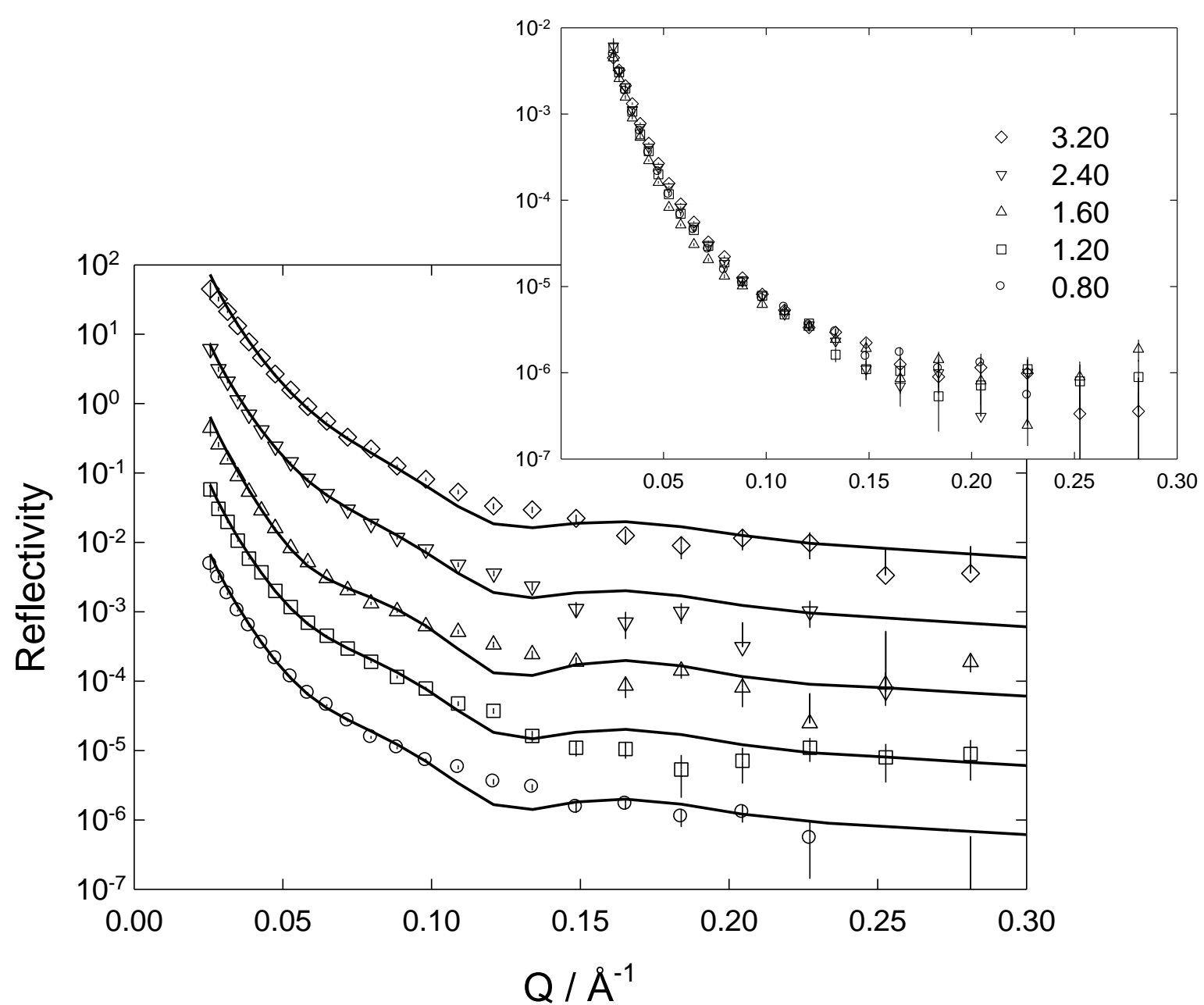

Figure 8. Reflectivity profiles for a series of d-ACE16 and h-PA spread amount (Contrast 5) at the CMSi oil- $\mathrm{D}_{2} \mathrm{O}$ interface. Solid lines correspond to the fit to the data, the fitted parameters are given in Table 10. Profiles are shifted by a factor of $\times \mathbf{1 0}$ for the purpose of clarity. The un-shifted profiles are shown in figure insert to highlight the lack of significant differences. Labels for the spread amount are in figure insert (units: $\times 10^{-6} \mathrm{~mol} \mathrm{~m}^{-2}$ ).

Table 10. Fitted parameters for the reflectivity profiles for Contrast 5 (Figure 8).

\begin{tabular}{|c|c|c|c|c|c|c|c|}
\hline $\begin{array}{l}\text { Spread amount } \\
\times 10^{-6} \mathrm{~mol} \mathrm{~m}^{-2} \\
\end{array}$ & & 0.80 & 1.20 & 1.60 & 2.40 & 3.20 & \\
\hline & $d / \AA( \pm 2)$ & \multicolumn{5}{|c|}{$\mathrm{Nb} \times 10^{-6} / \AA^{-2}$} & Roughness / §̊ \\
\hline Oil & I & \multicolumn{5}{|c|}{2.07} & I \\
\hline Layer 1 & 28 & 4.6 & 4.66 & 4.33 & 4.73 & 4.73 & 5.0 \\
\hline Layer 2 & 42 & 5.78 & 5.77 & 5.61 & 5.89 & 5.96 & 5.0 \\
\hline $\mathrm{D}_{2} \mathrm{O}$ & I & \multicolumn{5}{|c|}{6.35} & 5.0 \\
\hline
\end{tabular}




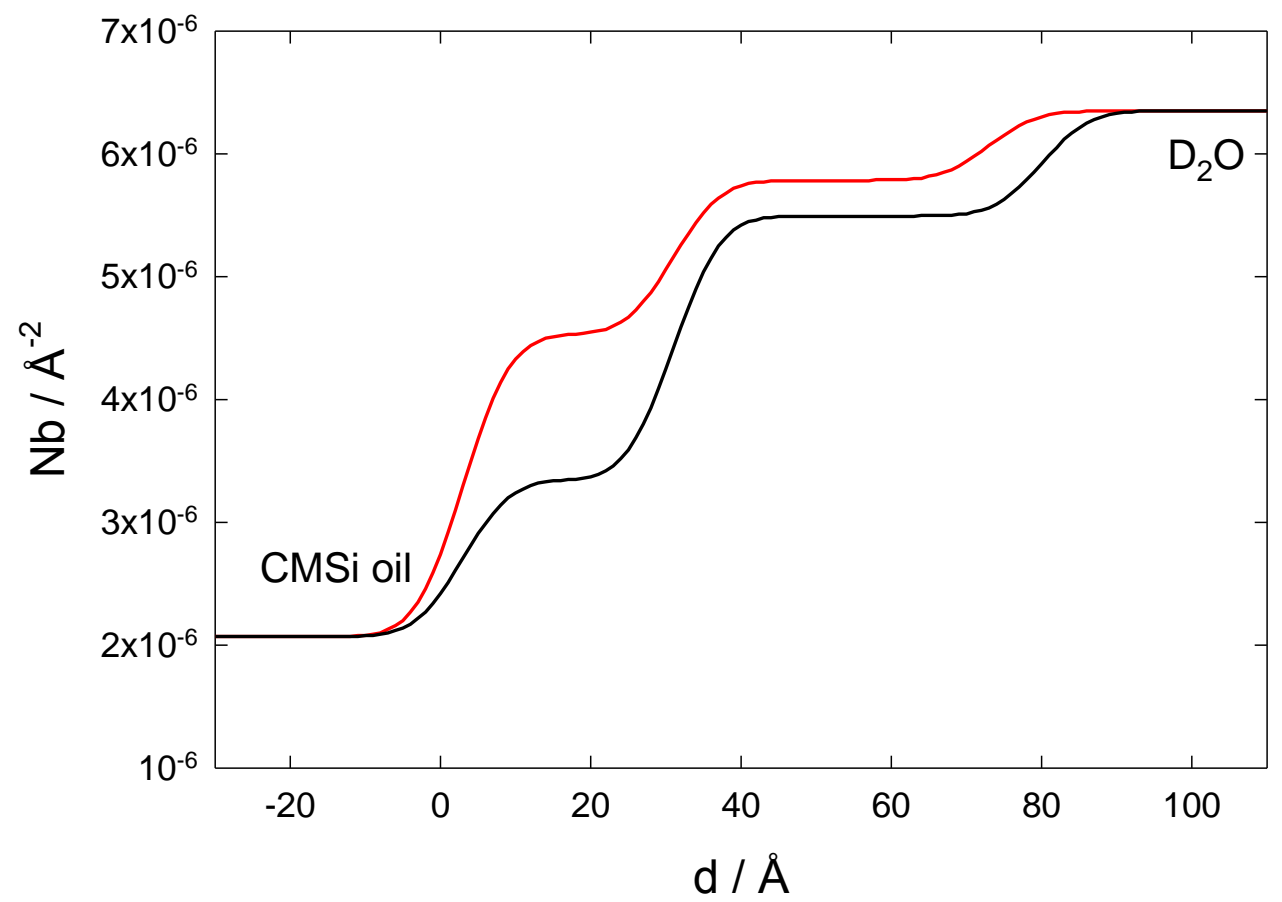

Figure 9. $N b$ profiles for Contrast 4 (black line) and Contrast 5 (red line). Given the small differences observed within the profiles for the individual contrasts, for each contrast only the average $N b$ values are shown.

\section{DISCUSSION}

The adsorbed amount for palmitic acid at the oil-water interface is shown in Figure 6(b) by the filled circles. For the spread amount equal $0.80 \times 10^{-6} \mathrm{~mol} \mathrm{~m}^{-2}$ the adsorbed amount is slightly higher than the maximum theoretical value; the difference is, however, very subtle. For all higher values of spread amount, the adsorbed amount is either equal to or lower than expected. No significant changes are observed with increasing the spread amount, suggesting that for PA the full coverage at the oil-water interface is already reached at the lowest spread amount. This full coverage of PA at the oil-water interface is reached at rather low values of adsorbed amount $\left(\Gamma<1 \times 10^{-6} \mathrm{~mol} \mathrm{~m}^{-2}\right)$, significantly lower than those observed for fatty acids (stearic acid) at the air-water interface [17]. The solubility of PA in water is negligible, whereas it has significant solubility in the oil phase. This suggests that the excess fatty acid might simply dissolve in the bulk oil phase. The adsorbed amount of PA 
drops even further when ACE16 is co-adsorbed at the interface. This is shown also in Figure 6(b), open circles. Again, very small changes are observed with increasing spread amount.

The adsorbed amount for ACE16 in the absence of PA (Figure 6(a)) shows an increase with spread amount up to $2.2 \times 10^{-6} \mathrm{~mol} \mathrm{~m}^{-2}$. The layer gets depleted as the spread amount increases beyond this point. In presence of PA, however, the adsorbed amount for ACE16 remains remarkably constant with increasing spread amount (yet smaller than in its absence). The fact that the adsorbed amount of both species at the oil-water interface is independent from the spread amount could be indicative of some ordering at the interface. Unfortunately, since neutron reflectivity is only sensitive to the $\mathrm{Nb}$ profile normal to the interface averaged over the interfacial plane, any such ordering cannot be detected using the current setting. The association of PA and ACE16 results in a monolayer of constant composition at the oil-water interface as a function of increasing spread amount. This result suggests the presence of a self-regulatory mechanism for the composition of the mixed adsorbed layer.

It is worth mentioning that at the lowest spread amount of the PA/ACE16 mixture $\left(0.80 \times 10^{-}\right.$ ${ }^{6} \mathrm{~mol} \mathrm{~m}{ }^{-2}$ of each component), the spread amount and adsorbed amount for ACE16 correspond well within error, thus indicating that ACE16 is entirely retained at the interface. On the other hand, the adsorbed amount for PA is extremely low even at the highest spread amount. In order to speculate about where the missing material from the interface resides, we should recall the behaviour of such mixed monolayers at the air-water interface. It has been suggested previously that ACE16 slowly dissolves from the water surface into the bulk aqueous phase [7]. Our recent experiment at the air water interface clearly showed how the presence of PA is beneficial from the point of view of retaining ACE16 at the surface [8]. It is then very likely that the dissolution of ACE16 into the water phase in presence of PA is also slowed down at the oil-water interface. Nevertheless, whereas the solubility of ACE16 in water is very small and that of PA is negligible, both species are readily soluble in hexadecane. Hence, even if only little material may dissolve into the aqueous sub-phase (as reported at the air-water interface), it seems more likely that most of the adsorbed material leaves the interface by dissolving into the oil phase.

The small amount of PA retained at the oil-water interface stems probably from the fact that it readily form dimers when present in organic solutions. The driving force for the formation of these dimers is the fact that carboxylate groups are both good donors and acceptors of hydrogen bonds [18]. Dimerisation effectively shields the hydrophilic parts of PA, thus rendering it more oil-soluble and more prone to desorb from the interface. Analogously, 
formation of PA/ACE16 interfacial complexes shields the hydrophilic parts of both molecules, by forming an H-bond between nitrogen atoms of ACE and carboxylate group of the PA [19]. As a result, the interfacial complex formed is more hydrophobic than the individual components and desorbs from the oil-water interface. While the fact that the azacrown ether and fatty acid do co-adsorb at the oil-water interface was evident from previous interfacial tension and surface rheology studies [20,21], the current experiment sheds new light on both the composition and surface activity of this complex. The adsorbed amount of PA in the mixed monolayer is very little and most of the material dissolves into the oil phase. However, the presence of PA at the interface significantly affects the adsorption profile of ACE16 (Figure 6(a)). The present results show that the fatty acid may have a regulatory effect on the surface concentration of ACE16.

The maximum adsorbed amount for both species seems to be already reached at spread amount $0.80 \times 10^{-6} \mathrm{~mol} \mathrm{~m}^{-2}$. At this surface coverage ACE16 is entirely retained at the interface, while most of the PA dissolves in the oil phase. As the surface coverage increases up to $1.60 \times 10^{-6} \mathrm{~mol} \mathrm{~m}^{-2}$, no significant changes are observed in the adsorbed amount of both species. The presence of PA seems to enhance the adsorbed amount of ACE16, which would be lower in its absence [7]. At spread amounts higher than $1.60 \times 10^{-6} \mathrm{~mol} \mathrm{~m}^{-2}$, although no changes are observed in the adsorbed amount for the individual species, the presence of PA reduces the adsorbed amount of ACE16.

The simultaneous analysis of Contrast 4 and Contrast 5 reflectivity data, with $\mathrm{D}_{2} \mathrm{O}$ as subphase, confirms previous observations that at the oil-water interface the interfacial region is much broader compared to the air-water interface. The $\triangle N b$ between the layers observed when moving from Contrast 4 to Contrast 5 must be attributed to the substitution of h-ACE16 with d-ACE16; hence the integration of the area comprised between the two $\mathrm{Nb}$ profiles in Figure 9 can be used to obtain a rough estimation of the adsorbed amount of ACE16 at the interface. The integrated area leads to an adsorbed amount $\Gamma \sim 1.1 \times 10^{-6} \mathrm{~mol} \mathrm{~m}^{-2}$, which is slightly higher than the values calculated simultaneously using the first three profiles. Because of calculations being carried out using the average values between all the fitted reflectivity profiles and because of the presence of interfacial roughness in the current model, we did not expect to obtain exactly the same values calculated using the first three contrasts. The $N b$ profiles in Figure 9 show that there is a significant $\Delta N b$ in the first layer, adjacent to the aqueous phase, when moving from Contrast 4 to Contrast 5 . The $\Delta N b$ is much less pronounced in the second layer. This suggests that most of the ACE16 is present in the first 
layer, where it forms a rough monolayer with the tail groups in contact with the oil phase. The thickness of the layer is $d=28 \AA$, more than a fully extended ACE16 molecule ( $21.7 \AA$ ), suggesting a staggered conformation of the adsorbed layer. The layer in contact with the aqueous phase contains less ACE16 than the oil-side layer, as can be seen by the smaller $\triangle N b$ in the profiles in Figure 9. The thickness, about twice the extended C-16 length, indicates the diffuse nature of the layer. The thickness of this diffuse layer was found to be slightly different between the two contrasts: it decreases from $49 \AA$ for Contrast 4 to $42 \AA$ for Contrast 5. In Contrast 5 the h-ACE16 was replaced by d-ACE16, whereas h-PA was used for both contrasts. The different layer thickness could be indicative of non-homogeneous distribution of the two components throughout the diffuse layer. At present we are not able to speculate with regard to the exact distribution of the individual components in the diffuse layer. However, given the thicker layer and the rise in $\mathrm{Nb}$ observed in Contrast 5, where ACE16 is deuterated, we believe ACE16 diffuses more toward the aqueous phase, while PA remains confined to the inner part of the interface.

The structure of d-ACE16 monolayer in absence of PA at the hexadecane-water interface was previously described and a two layer model was deployed to represent the interfacial structure. The oil side of the interface showed a compact monolayer, with thickness slightly smaller than a fully extended molecule. A loosely aggregated, diffuse monolayer was found on the aqueous side of the interface and its presence suggested that depletion of ACE16 from the interface occurs by dissolution in the aqueous phase. The decrease in $N b$ in the layer in contact with the oil phase, representing the compact ACE16 monolayer, is indicative of a decrease in the volume fraction of d-ACE16 and/or $\mathrm{D}_{2} \mathrm{O}$ (only an increase in volume fraction of CMSi oil would justify a decrease in $N b$ ). The $N b$ profiles as a function of d-ACE16 spread amount at the CMSi oil- $\mathrm{D}_{2} \mathrm{O}$ interface are shown in Figure 10 by the black lines. The arrow indicates the decrease in $N b$ in the layer in contact with the oil phase as a function of increasing spread amount. The red line in Figure 10 represents the $\mathrm{Nb}$ profile for Contrast 5 and is the same as reported in Figure 9. 


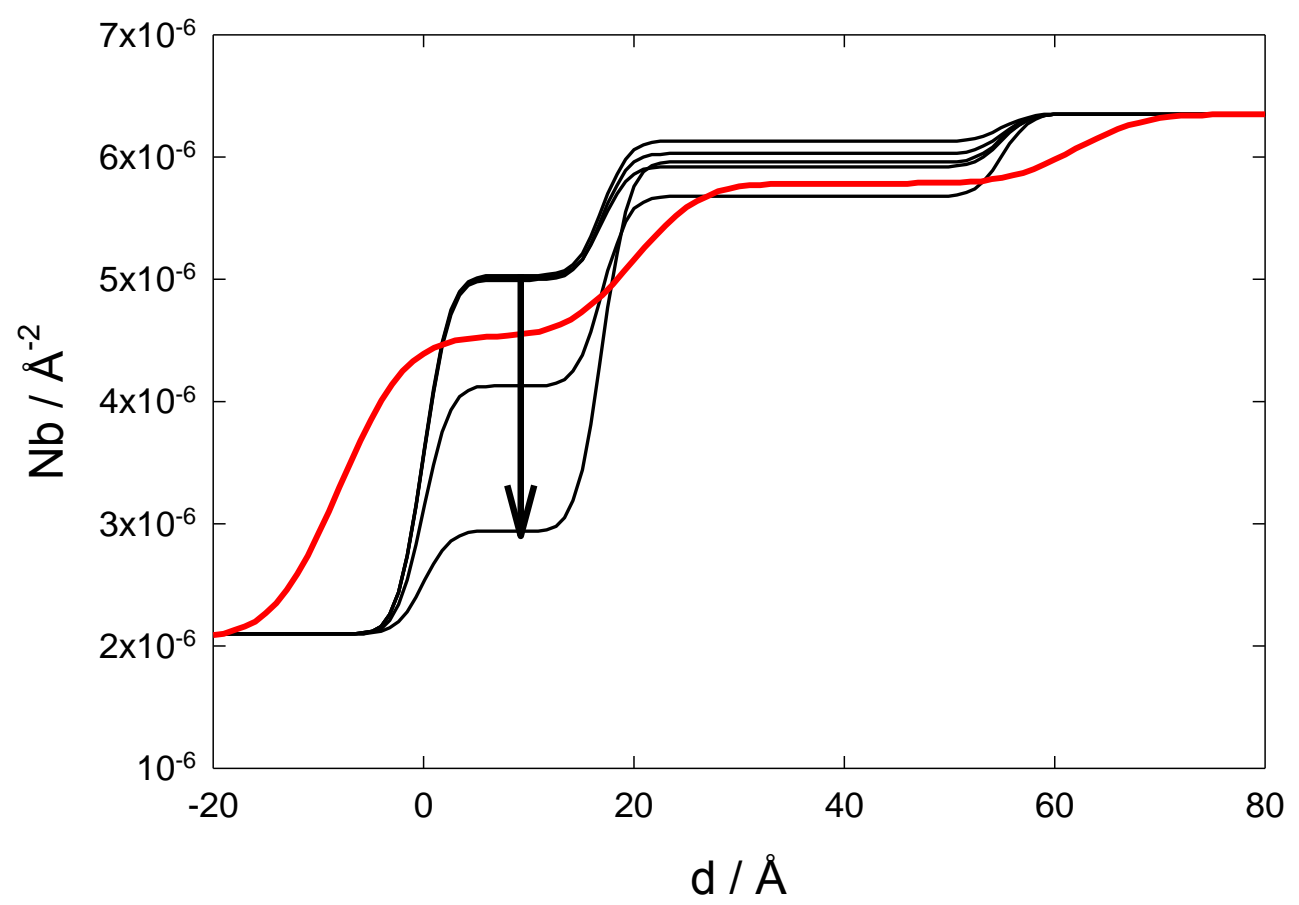

Figure 10. $N b$ profiles for $\mathrm{d}-\mathrm{ACE} 16$ at the $\mathrm{CMSi}-\mathrm{D}_{2} \mathrm{O}$ interface (the black lines), taken from a previous experiment [7]. The arrow indicates the increase in spread amount. The $\mathrm{Nb}$ profile for Contrast 5, reported from Figure 9, is also shown (red line). The latter profile is shifted so that the boundary between the two layers coincides with that for ACE16 alone (black lines).

The conformation of ACE16 at the oil-water interface in the presence (red line) and in the absence (black lines) of PA is remarkably similar. The only noticeable difference between the two data sets is the layer thickness, which increases when PA is co-adsorbed at the interface ( $d \sim 55 \AA$ for ACE16 alone, $d \sim 77 \AA$ for ACE16 and PA mixtures). The increase in overall layer thickness could be an indication of a dynamic interface when ACE16 and PA are coadsorbed at the interface, as opposed to a more static one when ACE16 is the only specie adsorbed.

The invariable adsorbed amount observed for both ACE16 and PA suggests that the interface rapidly reaches saturation at low surface coverage and the excess material is ejected into the bulk phase. Hence, two scenarios are possible:

(i) The excess material in the bulk phase does not interact with the adsorbed material and the dissolved material does not exchange with the interface.

(ii) The co-adsorption process at the oil-water interface is driven by equilibrium. The excess material which resides in the bulk phase exchanges with that in the adsorbed layer, leading to a dynamic interface. 
This ACE-fatty acid mixtures have been successfully used for metal ion transport in permeation liquid membrane systems (PLM); hence a static interface does not seem to be compatible with the efficient transport mechanism. We believe that the second scenario, whereby equilibrium between the material in solution in the oil phase and that adsorbed at the interface is established, is more compatible with the transport mechanism. In fact, easy exchange of both ACE16 and PA between the bulk and the interface would be desirable in a PLM device, where the extraction process takes place at the interfacial region. A confirmation for the more dynamic nature of the interface comes from the increase in overall layer thickness of the interfacial area observed when PA and ACE16 are co-adsorbed at the interface (Figure 10). Exchange of material between the interface and the bulk phase would in fact lead to an effective thickening of the interfacial region.

The macroscopic mechanism of transport across the membrane has already been discussed [22]; however the mechanism for the formation of the metal-carrier complex at the interface is still under dispute. We tentatively suggest here that, because of the very small adsorbed amount of PA at the oil-water interface, it is more likely that ACE16 interacts with the metal ion from the aqueous phase in the first place. As soon as this interfacial process is completed, the transport through the bulk of the membrane can eventually proceed via "paddlewheel" formation in the bulk organic (membrane) phase, where the fatty acid plays a crucial role [22].

\section{CONCLUSION}

Mixed monolayers of ACE16 and palmitic acid at the oil-water interface showed a remarkable uniformity in composition with increasing spread amount, where saturation of the interface was already achieved with low spread amount. A very little amount of palmitic acid is retained at the interface and it does not change with increasing spread amount. The excess material accommodates in the oil phase, playing an important role in equilibrating the interfacial concentration of ACE16. In the absence of PA the adsorbed amount for ACE16 increases up to a spread amount of about $2.5 \times 10^{-6} \mathrm{~mol} \mathrm{~m}^{-2}$ (see Figure 6 (a)). The presence of PA increases the surface concentration of ACE16 at low spread amount, but facilitates its dissolution into the oil phase at the high spread amount.

The structure of the mixed layer is rougher and thicker than a pure ACE16 layer, suggesting the instauration of a dynamic exchange between the bulk phase and the interface. Such 
exchange ensures a continuous turnover which reflects in more metal ions transported through the interface and increased efficiency of the PLM device.

The studies have been performed in the absence of metal ions, whereas in a real device a small concentration of metal ions would be present in the aqueous phases. The presence of ions would not excessively alter the conformation of the adsorbed layer at the air-water interface (extraction is prevented by the lack of a hydrophobic solvent) but may significantly affect the conformation of the adsorbed layer at the oil-water interface. As last step in the characterisation of these PLM devices, we are currently studying the effect of addition of metal ions such as $\mathrm{Cu}^{2+}$ to the bulk aqueous sub-phase.

\section{AUTHOR INFORMATION}

\section{Corresponding Author}

E-mail: a.zarbakhsh@qmul.ac.uk and kamil.wojciechowski@ch.pw.edu.pl

\section{ACKNOWLEDGMENT}

The authors wish to thank the Institute Laue-Langevin, for granting beam-time on the Figaro Reflectometer and the STFC (ISIS), UK, for part funding of a PhD studentship. This work was also partly funded by Warsaw University of Technology. 


\section{REFERENCES}

[1] M. Martino, M., Turner, A., Nimmo, M., Mar Chem, 2004, 88, 161.

[2] Buffle, J.; Parthasarathy, N.; Djane, N. K.; Matthiasson, L. Permeation Liquid

Membranes for Field Analysis and Speciation of Trace Compounds in Waters. IUPAC Series on Analytical and Physical Chemistry of Environmental Systems 2000, 6, 407.

[3] "Development of permeation liquid membrane systems for in situ trace metal speciation in water.",Salaun, P., Parthasarathy,N., Lagger,G., Martin, M., Buffle, J., Thiebaud, P., Koudelka, M., Van Leeuwen, H. P., Abstracts of Papers of the Am Chem. Soc. 220: 137ENVR Part 1, 2000.

[4] Wojciechowski, K.; Buffle, J. and Miller, R, Colloids and Surfaces A: Physicochem. Eng. Aspects, 2005, 261, 49.

[5] Wojciechowski K., Brzozowska A.; J. Phys. Chem. C, 2012, 116, 12584.

[6] Wojciechowski K., Gutberlet T., Tikhonov A., Kashimoto K., Schlossman M.; Chem Phys Lett, 2010, 487, 62.

[7] Zarbakhsh, A; Webster, J.R.P and Wojciechowski, K, Langmuir, 2009, 25, 11569.

[8] Zarbakhsh A., Campana M., Webster J. R. P., Wojciechowski K.; Langmuir, 2010, 26, 18194.

[9] Salaun P., Bujard F., Berdondini L., Koudelka-Hep M., Buffle J.; Electroanal., 2004, 16, 811.

[10] BDG Synthesis, New Zealand, www.bdg.co.nz.

[11] Campbell, R. A., Wacklin, H. P., Sutton, I., Cubitt, R., and Fragneto, G., The European Physical Journal Plus, Volume 126, Number 11, 107, 2011.

[12] Campana M, Webster J. R. P., Lawrence M. J., Zarbakhsh A.; Soft Matter, 2012, 8, 8904.

[13] Lu J. R., Thomas R. K., Penfold J.; Adv. Colloid Interface Sci., 2000, 84, 143. 
[14] Zarbakhsh A., Querol A., Bowers J., Yaseen M., Lu J. R., Webster J. R. P., Langmuir, 2005, 21, 11704.

[15] Dickinson E., Horne D. S., Richardson R. M.; Food Hydrocolloid, 1993, 7, 497.

[16] Born M., Wolf E. in Principles of optics; Peragamon: Oxford, 1980.

[17] Qi S., Roser S. J., Deutsch D., Barker S. A., Craig D. Q. M.; J. Pharm. Sci., 2008, 97, 1864.

[18] Brzozowska I., Figaszewski Z. A., Colloid Surface B, 2003, 30, 187.

[19] Wojciechowski, K.; Buffle, J.; Biosens Bioelectron, 2004, 20, 1051.

[20] Wojciechowski K., Buffle J., Miller R.; Colloids Surf A, 2005, 261, 49.

[21] Wojciechowski K., Buffle J., Miller R.; Coll. Surf. A, 2007, 298, 63.

[22] Wojciechowski K., Kucharek M., Buffle J.; J Membr Sci, 2008, 314, 152. 
Supporting Information

Contrast 1 and Contrast 3 were fitted using a single layer model, whereas a two layer model was used for Contrast 2. Such a two layer model can adequately represent also the reflectivity profiles for Contrast 1 and 3. As an example, the reflectivity profiles for spread amount $1.60 \times 10^{-6} \mathrm{~mol} \mathrm{~m}^{-2}$ are shown in Figure (a) (Contrast 1) and Figure (b) (Contrast 3) using a two layer model.

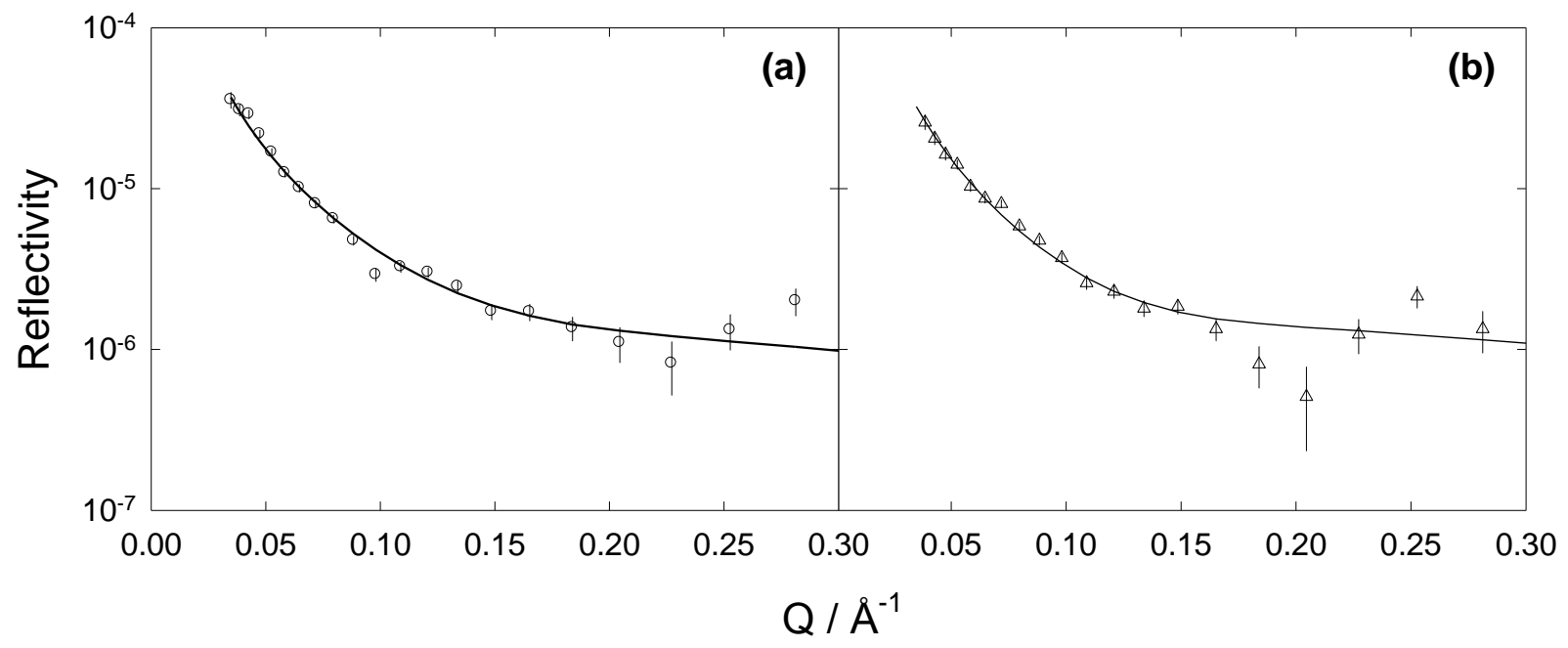

Transposition of the two layer model adopted for Contrast 2 to the other two contrasts. Only one spread amount is shown $\left(1.60 \times 10^{-6} \mathrm{~mol} \mathrm{~m}^{-2}\right)$. (a): Contrast 1 , (o); (b):

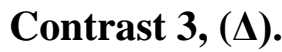




\section{Graphical abstract}

\section{Surfactant Mixtures at the Oil-Water Interface}

Mario Campana ${ }^{1}$, John. R. P. Webster ${ }^{2}$, Thomas Gutberlet ${ }^{3}$, Kamil Wojciechowski ${ }^{4^{*}}$ and Ali Zarbakhsh ${ }^{1 *}$

${ }^{1}$ School of Biological \& Chemical Sciences, Queen Mary, University of London, Joseph Priestley Building, Mile End Road, London E1 4NS, United Kingdom.

${ }^{2}$ ISIS Neutron Facility, Science and Technology Facilities Council, Rutherford Appleton Laboratory, Harwell Science and Innovation Campus, Didcot, OX11 0QX, United Kingdom.

${ }^{3}$ Helmholtz-Zentrum Berlin für Materialien und Energie GmbH, Hahn-Meitner-Platz 1, D-14109 Berlin, Germany.

${ }^{4}$ Warsaw University of Technology, Faculty of Chemistry, Noakowskiego 3, 00-664 Warsaw, Poland.

*Authors to whom correspondence should be addressed. Email: a.zarbakhsh@qmul.ac.uk and kamil.wojciechowski@ch.pw.edu.pl

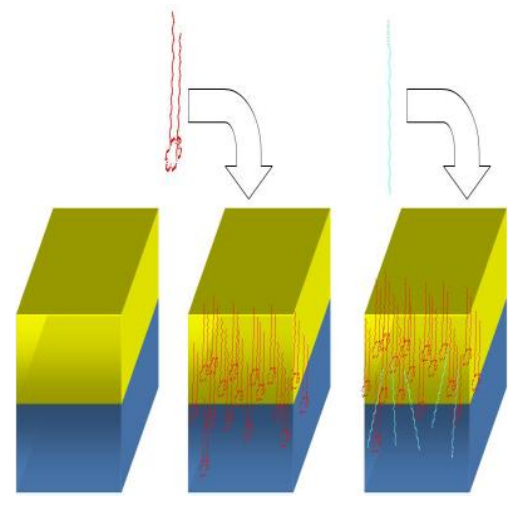

